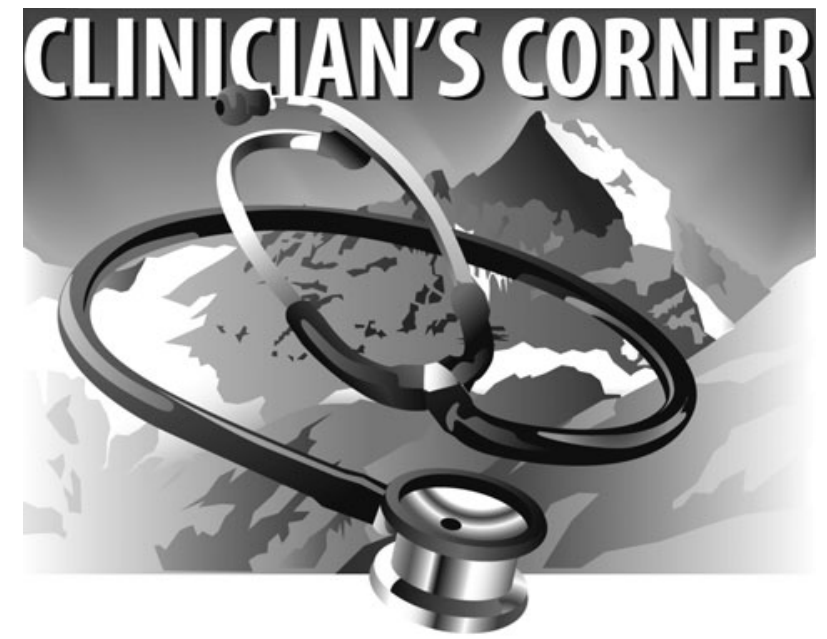

\title{
Considerations on Safety and Treatment of Patients with Chronic Heart Failure at High Altitude
}

\author{
Piergiuseppe Agostoni, $\mathrm{MD}, \mathrm{PhD}^{1-3}$
}

\begin{abstract}
Piergiuseppe Agostoni. Considerations on safety and treatment of patients with chronic heart failure at high altitude. High Alt Med Biol 14:96-100, 2013.-Prognosis and quality of life of chronic heart failure (HF) patients have greatly improved over the last decade. Consequently, many patients are willing to spend leisure time at altitude, usually $<3500 \mathrm{~m}$, but their safety in doing so is undefined. HF is a syndrome that often has relevant comorbidities, such as pulmonary hypertension, COPD, unstable cardiac ischemia, and anemia. HF co-morbidities may per se impede a safe stay at altitude. Exercise at simulated altitude is associated with a reduction in performance, which is greater in HF patients than in normal subjects and greater in patients with most severe HF. In normal subjects, the reduction in performance is $\sim 2 \%$ every $1000 \mathrm{~m}$ altitude increase, whereas it is $4 \%$ and $10 \%$ in HF patients with normal or slightly diminished exercise capacity and in HF patients with markedly diminished exercise capacity. On-field experience with HF patients at altitude is limited to subjects driven to altitude ( $3454 \mathrm{~m})$ for a few hours. The data showed a reduction in exercise capacity similar to that reported at simulated altitude. "Optimal" HF treatment in patients spending time at altitude is likely different from optimal treatment at sea level, particularly as regards $\beta$-blockers. Carvedilol, a $\beta_{1}-\beta_{2}-\alpha$-blocker, reduces the hypoxic ventilatory response through a reduction of the chemoreflex response, and it reduces alveolar-capillary gas diffusion, which is under control by $\beta_{2}$-receptors. These actions are not shared by selective $\beta_{1}$-blockers such as bisoprolol and nebivolol, which should be preferred for treatment of HF patients willing to spend time at altitude. In conclusion, spending time at altitude $(<3500 \mathrm{~m})$ is safe for HF patients, provided that subjects are free of co-morbidities that may directly interfere with the adaptation to altitude. However, HF patients experience a reduction of exercise capacity in proportion to HF severity and altitude. Finally, HF patients should undergo a specific "altitude-tailored treatment" to avoid pharmacological interference with altitude adaptation mechanisms.
\end{abstract}

\section{Introduction}

C HRONIC HEART FAILURE (HF) is among the most frequent diseases in western countries, with 670,000 new cases a year in the US (Lloyd-Jones et al., 2010). Both survival and quality of life of HF patients significantly improved in the last decades, mainly due to an improvement in therapies. Con- sequently, many HF patients have a normal or almost normal life for a prolonged time, which may include the chance of spending leisure time at altitude. This usually means an altitude between 1000 and 3500 m.Questions that are often not easy to answer are whether staying at high altitude is safe for HF patients, whether all patients behave in the same way at altitude, or whether there are differences among patients at

${ }^{1}$ Centro Cardiologico Monzino, IRCCS, Milan, Italy; ${ }^{2}$ Dipartimento di scienze cliniche e di comunità, Università di Milano, Milan Italy; ${ }^{3}$ Division of Pulmonary and Critical Care Medicine, Department of Medicine, University of Washington, Seattle, Washington. 
altitude with respect to HF severity and co-morbidities, and, finally, whether HF patients should adapt their physical performance at altitude. Moreover, it is unclear whether, among the different HF therapeutic strategies available, there is one most appropriate for subjects who plan an altitude sojourn.

Chronic HF is a syndrome which is characterized by the impairment of several body parts, such as lungs, kidney, muscles, pulmonary circulation, red blood cells, and sympathetic nervous system, all of which imply a specific adaptation to altitude or may be further impaired at altitude. Moreover, HF patients frequently have co-morbidities, such as-just to mention some-lung diseases, systemic hypertension, metabolic syndrome, peripheral and coronary vascular diseases, all of which may have a detrimental role in altitude adaptation, particularly when combined with HF. Therefore, patients with HF and lung disease, pulmonary hypertension-including out-of-proportion pulmonary hypertension due to $\mathrm{HF}$ (Simonneau et al., 2009)—anemia, severe renal insufficiency, angina, or primitive cardiac valvular or pericardial diseases, who are willing to ascend to altitude, should be evaluated for HF and also for the specific HF co-morbidities they are affected by. For instance, in a patient with HF and COPD, the latter is the factor that actually limits altitude exposure due to a greater arterial $\mathrm{Po}_{2}$ reduction compared to non-COPD HF subjects (Gong et al., 1984; Gong, 1989). A counterproof of this is the observation that, among HF patients exposed to low altitude (below sea level as in the Dead Sea), patients with HF and COPD (with exerciseinduced arterial desaturation) improve exercise performance more than HF patients without COPD (Abinader et al., 1999).

Several physiological adaptations to or consequences of high altitude exposure may, in principle, negatively influence the physical condition of HF patients, which includes the increase of sympathetic activity, pulmonary and systemic blood pressure, heart rate, lung fluid content, or the reduction of stroke volume (Agostoni et al., 2009; Cogo and Miserocchi, 2011; Rimoldi et al., 2010; Swenson, 2011). Some of these effects are limited in time but, because they are possibly associated with a deterioration of HF, they should be cautiously considered when evaluating whether a HF patient can go to high altitude. Therefore, at first glance, HF patients should be advised to not go to altitude, albeit it has been very recently suggested that repeated, short-lasting (3-4 hours) exposures to a simulated altitude up to $2700 \mathrm{~m}$ may be beneficial for HF patients in terms of quality of life, muscular strength, and exercise performance (Saeed et al., 2012). However, a 3-4-hour exposure may be different from a 24-hour or longer exposure, because the negative effects of some adaptations to high altitude may need more time to develop completely.

Very few non-laboratory, real-life experiences are presently available for HF patients at altitude. Indeed, albeit laboratory studies are able to mimic hypoxic conditions, this is not the case for other variables encountered at altitude, such as a cold and dry environment, as well as poor weather conditions in general. Exercising in a cold and dry environment does imply a greater energy consumption if compared to exercise performed in a comfortable setting. However, it is now appreciated that patients with coronary artery disease and preserved left ventricular function can safely reach altitude and exercise there, and that adverse cardiac events, such as unstable angina or coronary syndrome, do not occur more frequently than at sea level if subjects unaccustomed to exercise are excluded (Dehnert and Bartsch, 2010; Schmid et al., 2006; de
Vries et al., 2010). Indeed, Schmid et al. (2006) showed that coronary patients who have been completely revascularized can safely go and exercise at the Jungfraujoch (3454 m), and de Vries et al. (2010) showed that patients with a history of myocardial infarction and preserved left ventricular function presented a decrease in exercise capacity similar to that of healthy controls at $4200 \mathrm{~m}$ in the Aconcagua region after a 10-day acclimatization (de Vries et al., 2010). No data are available for HF patients, except for another study of the Schmid group (Nobel et al., 2010), which showed that HF patients in stable clinical condition, class NYHA II, and with peak $\mathrm{Vo}_{2}$ at $540 \mathrm{~m}>50 \%$ of that predicted, can safely reach and exercise at the Jungfraujoch $(3454 \mathrm{~m})$. In these HF patients, peak $\mathrm{Vo}_{2}$ decreased by $22 \%$ at altitude, and no significant changes were observed in the arrhythmic pattern as well as in echocardiographic measurements, except for an increase in pulmonary pressure. However, it should be noticed that, in the study by Schmid, HF patients reached the Jungfraujoch by cable car and remained at $3454 \mathrm{~m}$ for only few hours. Both the absence of a significant effort to reach altitude and the short-lasting altitude exposure may have contributed to the positive results reported by Schmid. As a matter of fact, a significant effort is associated to lung fluid accumulation as a few day stay at altitude is (Agostoni et al., 2009; Heath and Williams, 1981; Singh et al., 1965).

Several HF patients have implanted defibrillators (ICD) for arrhythmia treatment, and several have pacemaker-mediated resynchronization therapy (CRT) for HF treatment. At present, little information exists regarding function of ICD and CRT at altitude. Weilenmaen et al. (2000) studied 13 patients with single chamber pacemakers and found no changes in ventricular stimulation thresholds at a simulated altitude of $4000 \mathrm{~m}$, although the duration of exposure was only $30 \mathrm{~min}$ and may not accurately reflect what would happen with longer stays in hypobaric hypoxia. Moreover, in a recent survey study in Swiss patients living at altitude, it has been reported that ICD shock was rare (4\%) and it has been suggested that ICD patients living at moderate altitude may safely perform moderate physical activity (Kobza et al., 2008).

More precise information is available regarding exercise performance in HF patients during exposure to acute hypoxia as obtainable in a laboratory setting. Indeed, exercise performance was progressively reduced in HF patients if they exercised at a simulated altitude of 1000, 1500, 2000, and $3000 \mathrm{~m}$ (Fig. 1). Notably, this reduction was greater in HF patients than in normal subjects, and it was greater in the more severe HF patients (Agostoni et al., 2000), where the severity of the disease was defined by peak $\mathrm{Vo}_{2}$ (normal exercise performance: peak $\mathrm{Vo}_{2}>20 \mathrm{~mL} / \mathrm{min} / \mathrm{kg}$, slightly diminished exercise capacity: peak $\mathrm{VO}_{2}$ between 20 and $15 \mathrm{~mL} / \mathrm{min} / \mathrm{kg}$, and markedly diminished exercise capacity: peak $\mathrm{Vo}_{2}<15 \mathrm{~mL} /$ $\mathrm{min} / \mathrm{kg}$ ). As an average, we observed an exercise capacity reduction of $\sim 2 \%, \sim 4 \%$, and $\sim 10 \%$ every $1000 \mathrm{~m}$ altitude increase in normal subjects, HF patients with normal or slightly diminished exercise capacity, and HF patients with markedly diminished exercise capacity, respectively. These data are in line with Schmid's findings at the Jungfraujoch (Nobel et al., 2010). Moreover, it is important to observe that alveolar capillary gas diffusion correlates with exercise performance in HF both at sea level (Agostoni et al., 2006a) and at simulated altitude (Agostoni et al., 2002b), and that alveolar capillary gas diffusion during exercise increases in healthy subjects, is unchanged in patients with moderate HF, and 


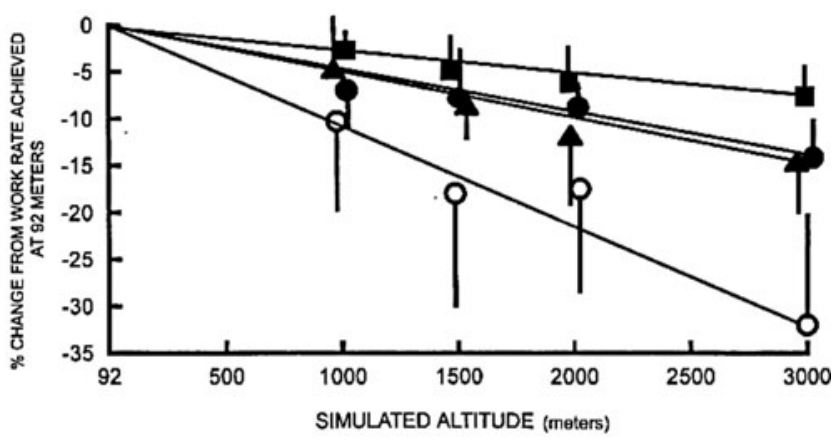

FIG. 1. Mean ( $\pm 95 \%$ confidence intervals) reduction in maximum work rate with simulated altitude, as a percentage decrease from maximum work rate at $92 \mathrm{~m}$. Slopes differed $(p<0.05)$ in healthy subjects $(-2 \% \pm 1 \%$ per $1,000 \mathrm{~m}$; filled squares) compared with patients, and between patients with normal $(-4 \pm 2 \%$; filled circles), or slightly diminished workload ( $-4 \pm 2 \%$; filled triangles) compared with patients with markedly diminished workload (-10 $\pm 3 \%$; open circles). From Agostoni et al., 2000.

decreases in patients with severe HF (Agostoni et al., 2003; Cattadori et al., 2009). Alveolar capillary gas diffusion negatively correlates with the widening of the alveolar-capillary $\mathrm{Po}_{2}$ gradient at peak exercise both in normoxic and hypoxic exercise (Agostoni et al., 2002b). Interestingly, HF patients who are able to increase alveolar capillary diffusion during light exercise are those who showed a lowest exercise performance reduction at a $2000 \mathrm{~m}$ simulated altitude (Fig. 2) (Agostoni et al., 2002b). The bulk of the above-reported information suggests that alveolar capillary gas diffusion at rest and its changes during exercise influence the exercise performance of HF patients in hypoxic conditions.

It should also be noticed that we have no data on the effects of prolonged altitude sojourns for HF patients. Indeed, altitude adaptation includes, in healthy subjects, among others, improvement of ventilation, alveolar capillary diffusion, and oxygen carrying capacity in the blood. All these should increase exercise performance of HF patients. Similarly we have no data to answer the frequently asked question on how fast a HF patient can safely travel to altitude, including rate of as-

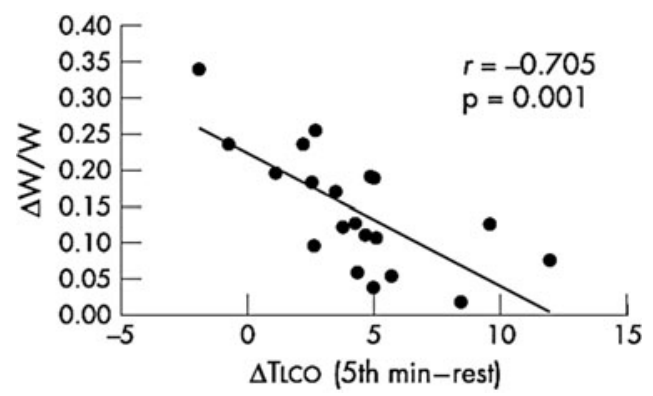

FIG. 2. Reduction of exercise capacity in hypoxia. $\Delta \mathrm{W} / \mathrm{W}=$ [maximum workload achieved in normoxia - maximum workload achieved in hypoxia]/maximum workload achieved in normoxia. $\triangle \mathrm{TLCO}=$ differences in lung diffusing capacity for carbon monoxide between the fifth minute of exercise and rest in heart failure patients. Patients with the greatest capability to increase TLCO during exercise are those who show the smallest reduction in exercise capacity in hypoxia. From Agostoni et al., 2002b. cent and staging at intermediate altitude. Consequently, cautious advice should be given to HF patiens willing to spend prolonged time at altitude.

All the above-reported data, either at simulated or at real altitude, have been obtained in HF patients in stable clinical conditions and on so-called optimal medical treatment. The latter is defined at sea level, but the optimal medical treatment for a given HF patient may be different at altitude. Two factors among several others that influence exercise performance at altitude, and precisely the alveolar capillary gas diffusion and the chemoreflex-mediated ventilatory response to hypoxia, are impaired in HF and, most importantly, they can be directly influenced by drugs used for HF treatment. Indeed, ion transport and the accompanying fluid movement across the alveolar capillary membrane are active phenomena under the control of $\beta_{2}$ receptors located on the airway surface of type I and II alveolar cells, and chemoreflex is regulated by angiotensine, nitric oxide, $\beta_{1}, \beta_{2}$ and $\alpha$ receptors. Angiotensine $1\left(\mathrm{AT}_{1}\right)$ receptor blockers and $\beta$-blockers are among the drugs used for the treatment of HF. The high-altitude adaptation of a normal subject on $\mathrm{AT}_{1}$ receptor blockers (telmisartan) is the main topic of an extensive research project, the HIGHCARE project, which was recently conducted at the Mount Everest South Base Camp (5400 m). No result on the effect of telmisartan on ventilatory and blood pressure control at high altitude has been released yet. Differently, it is now appreciated that the exercise performance of healthy subjects at high altitude is influenced by the type of $\beta$-blocker used. In a recent report, Valentini et al. (2011) showed that at Capanna Regina Margherita $(4560 \mathrm{~m})$ peak $\mathrm{Vo}_{2}$ was lower in healthy subjects treated with carvedilol $\left(\beta_{1}-\beta_{2}\right.$ receptor blocker) than in those on nebivolol (a selective $\beta_{1}$ receptor blocker). This difference was related to a greater peak exercise ventilation at altitude with nebivolol versus carvedilol. Finally, some preliminary data by our group on the effects of acetazolamide at high altitude indicate that this drug may be particularly useful at altitude in HF patients, albeit the opposite happens at sea level (Apostolo et al., 2008).Indeed, acetazolamide seems to prevent the lung fluid accumulation observed at altitude and to counteract altitude-induced lung diffusion reduction (Agostoni et al. 2013).

Some studies have been carried out by our group in Milan to assess the effects of HF treatment on exercise performance

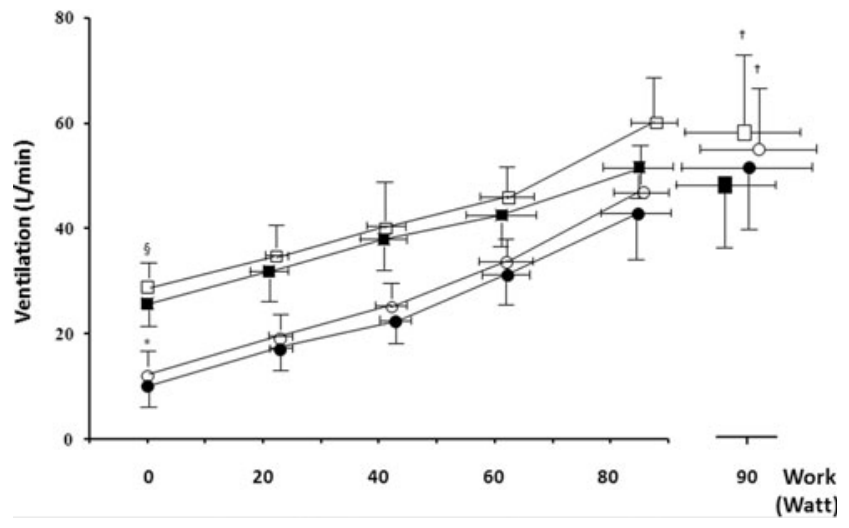

FIG. 3. Effect of carvedilol (filled symbols) versus placebo (empty symbols) on ventilation at different work rates, both in normoxic (circles) and in hypoxic (squares) conditions. Data from Agostoni et al., 2006b. 
of patients at a simulated altitude of $2000 \mathrm{~m}$ (Agostoni et al., 2002a; 2002b; 2007). This altitude was chosen because it is likely to be reached by HF patients during leisure time in the mountains. Two issues were analyzed: the effects of different $\beta$-blockers on alveolar capillary gas diffusion and those on the regulation of ventilation during exercise. As regards their effects on alveolar capillary gas diffusion, chronic carvedilol treatment is associated to a reduction of total alveolar capillary diffusion, as inferable from DLCO measurement (Agostoni et al., 2002a). By splitting DLCO into its two components, membrane diffusion and capillary volume, we showed that carvedilol reduced the former. We then showed that DLCO was reduced in HF patients on carvedilol, but not in those on bisoprolol (Agostoni et al., 2006b), and that this reduction directly correlated with a reduction of exercise performance in $\mathrm{HF}$ patients with DLCO $<80 \%$ of its predicted value (Agostoni et al., 2007). We very recently (Contini et al., 2012) confirmed this observation in a cross-over study comparing HF patients on carvedilol, bisoprolol, and nebivolol, the CARNEBI trial, and we showed that the reduction in DLCO was only observed during treatment with carvedilol. Interestingly, the reduction in DLCO was associated to an increase of the alveolar capillary $\mathrm{po}_{2}$ gradient, confirming the physiological significance of an even modest reduction in DLCO. Ventilation is regulated by several factors, including chemoreceptors. Chemoreceptor response in HF is increased as a part of the increased sympathetic tone, and this is one of the reasons why ventilation efficiency is reduced during exercise in HF. This reduction has a significant prognostic role (Gitt et al., 2002; Guazzi et al., 2008). Ventilation efficiency during exercise is usually assessed by the analysis of the ventilation to $\mathrm{VCO}_{2}$ relationship, which increases in case of inefficiency. Carvedilol reduces ventilation at a given work rate both in normoxic and hypoxic conditions (Fig. 3) (Agostoni et al., 2006b). This reduction is associated with a lower $\mathrm{PaO}_{2}$ (Agostoni et al., 2006b). This is not the case for bisoprolol and nebivolol (Agostoni et al., 2002a; 2010). Albeit a reduction of the ventilation to $\mathrm{VCO}_{2}$ relationship at sea level is likely to be a positive event, the opposite is probably true at altitude, being the ventilatory response necessary to counterbalance the effects of a reduced inspired $\mathrm{po}_{2}$. In the CARNEBI trial, we showed that carvedilol is the $\beta$-blocker that most reduces the chemoreceptor response to hypoxia and hypercapnia (both central and peripheral). The bulk of the above-reported studies indicates that a $\beta_{1}$ selective blocker, either bisoprolol or nebivolol, should be preferred to a $\beta_{1}-\beta_{2}$ blocker, such as carvedilol, for treating HF patients who are expected to spend time at altitude. It should be acknowledged, however, that we have no data as regards the effect of prolonged hypoxia exposure, as during a long sojourn at altitude, in HF patients with different betablocker treatments.

In conclusion, HF patients can safely spend leisure time at altitude up to $3500 \mathrm{~m}$, provided that they are on optimal, "altitude-tailored" HF treatment. HF patients, however, should expect a reduction of physical performance in relationship to the severity of the disease and to the altitude they will reach. Patients with HF co-morbidities should be evaluated with care, because the co-morbidities may definitively preclude HF patients from safely staying at altitude.

\section{Author Disclosure Statement}

No competing financial interests exist.

\section{References}

Abinader EG, Sharif DS, and Goldhammer E. (1999). Effects of low altitude on exercise performance in patients with congestive heart failure after healing of acute myocardial infarction. Am J Cardiol 83:383-387.

Agostoni P, Apostolo A, Cattadori G, et al. (2010). Effects of beta-blockers on ventilation efficiency in heart failure. Am Heart J 159:1067-1073.

Agostoni P, Bussotti M, Cattadori G, et al. (2006a). Gas diffusion and alveolar-capillary unit in chronic heart failure. Eur Heart J 27:2538-2543.

Agostoni P, Caldara G, Bussotti M, et al. (2009). Continuous positive airway pressure increases haemoglobin $\mathrm{O} 2$ saturation after acute but not prolonged altitude exposure. Eur Heart J 31:457-463.

Agostoni P, Cattadori G, Bianchi M, and Wasserman K. (2003). Exercise-induced pulmonary edema in heart failure. Circulation 108:2666-2671.

Agostoni P, Cattadori G, Guazzi M, et al. (2000). Effects of simulated altitude-induced hypoxia on exercise capacity in patients with chronic heart failure. Am J Med 109:450-455.

Agostoni P, Contini M, Cattadori G, et al. (2007). Lung function with carvedilol and bisoprolol in chronic heart failure: Is beta selectivity relevant? Eur J Heart Fail 9:827-833.

Agostoni P, Contini M, Magini A, et al. (2006b). Carvedilol reduces exercise-induced hyperventilation: A benefit in normoxia and a problem with hypoxia. Eur J Heart Fail 8:729-735.

Agostoni P, Guazzi M, Bussotti M, De Vita S, and Palermo P. (2002a). Carvedilol reduces the inappropriate increase of ventilation during exercise in heart failure patients. Chest 122:2062-2067.

Agostoni PG, Bussotti M, Palermo P, and Guazzi M. (2002b). Does lung diffusion impairment affect exercise capacity in patients with heart failure? Heart 88: 453-459.

Agostoni P, Swenson ER, Fumagalli R, et al. (2013). Acute high-altitude exposure reduces lung diffusion: Date from the HIGHCARE Alps project. Resp Physiol Neurobiol doi:pii: S1569-9048(13)00100-6. 10.1016/j.resp.2013.04.005. [Epub ahead of print 26 April 2013].

Apostolo A, Contini M, Antonioli L, et al. (2008). Effetti dell'acetazolamide sul respiro periodico durante esercizio nello scompenso cardiaco cronico. G Ital Cardiol 9:C158.

Cattadori G, Wasserman K, Meloni C, et al. (2009). Alveolar membrane conductance decreases as BNP increases during exercise in heart failure. Rationale for BNP in the evaluation of dyspnea. J Card Fail 15:136-144.

Cogo A, and Miserocchi G. (2011). Pro: Most climbers develop subclinical pulmonary interstitial edema. High Alt Med Biol 12:121-124; discussion 131-132.

Contini M, Paolillo S, Iorio A, et al. (2012). Comparison of carvedilol, nebivolol and bisoprolol on cardiopulmonary function in moderate heart failure. ESC Congress, abstract 86733.

de Vries ST, Komdeur P, Aalbersberg S, van Enst GC, Breeman $A$, and van 't Hof AW. (2010). Effects of altitude on exercise level and heart rate in patients with coronary artery disease and healthy controls. Neth Heart J 18:118-121.

Dehnert C, and Bartsch P. (2010). Can patients with coronary heart disease go to high altitude? High Alt Med Biol 11: 183-188.

Gitt AK, Wasserman K, Kilkowski C, et al. (2002). Exercise anaerobic threshold and ventilatory efficiency identify heart failure patients for high risk of early death. Circulation 106: 3079-3084.

Gong H, Jr. (1989). Advising patients with pulmonary diseases on air travel. Ann Intern Med 111:349-351. 
Gong H, Jr, Tashkin DP, Lee EY, and Simmons MS. (1984). Hypoxia-altitude simulation test. Evaluation of patients with chronic airway obstruction. Am Rev Respir Dis 130: 980-986.

Guazzi M, Myers J, Abella J, et al. (2008). The added prognostic value of ventilatory efficiency to the Weber classification system in patients with heart failure. Int J Cardiol 129: 86-92.

Heath D, and Williams DR. (1981). High Altitude Pulmonary Edema. Man at High Altitude. Edinburgh, Churchill Livingstone: $151-168$.

Kobza R, Duru F, and Erne P. (2008). Leisure-time activities of patients with ICDs: Findings of a survey with respect to sports activity, high altitude stays, and driving patterns. Pacing Clin Electrophysiol 31:845-849.

Lloyd-Jones D, Adams RJ, Brown TM, et al. (2010). Heart disease and stroke statistics-2010 update: A report from the American Heart Association. Circulation 121:e46-e215.

Nobel D, Novak J, Palau P, Trepp A, Saner H, and Schmid JP. (2010). Safety and tolerance of high altitude exposure (3454 m) in non-acclimatized patients with chronic heart failure. Eur J Cardiovasc Prev Rehabil 17:S67.

Rimoldi SF, Sartori C, Seiler C, et al. (2010). High-altitude exposure in patients with cardiovascular disease: Risk assessment and practical recommendations. Prog Cardiovasc Dis 52:512-524.

Saeed O, Bhatia V, Formica P, et al. (2012). Improved exercise performance and skeletal muscle strength after simulated altitude exposure: A novel approach for patients with chronic heart failure. J Card Fail 18:387-391.
Schmid JP, Noveanu M, Gaillet R, Hellige G, Wahl A, and Saner $H$. (2006). Safety and exercise tolerance of acute high altitude exposure $(3454 \mathrm{~m})$ among patients with coronary artery disease. Heart 92:921-925.

Simonneau G, Robbins IM, Beghetti M, et al. (2009). Updated clinical classification of pulmonary hypertension. J Am Coll Cardiol 54:S43-54.

Singh I, Kapila CC, Khanna PK, Nanda RB, and Rao BD. (1965). High-Altitude Pulmonary Oedema. Lancet 1:229-234.

Swenson ER. (2011). Con: Most climbers do not develop subclinical interstitial pulmonary edema. High Alt Med Biol 12:125-128; discussion 129-130.

Valentini M, Revera M, Bilo G, et al. (2011). Effects of beta-blockade on exercise performance at high altitude: A randomized, placebo-controlled trial comparing the efficacy of nebivolol versus carvedilol in healthy subjects. Cardiovasc Ther 30:240-248.

Weilenmann D, Duru F, Schonbeck M, et al. (2000). Influence of acute exposure to high altitude and hypoxemia on ventricular stimulation thresholds in pacemaker patients. Pacing Clin Electrophysiol 23:512-515.

Address correspondence to: Piergiuseppe Agostoni, MD, PhD Centro Cardiologico Monzino

via Parea 4 20138, Milano Italy E-mail: piergiuseppe.agostoni@unimi.it 